\title{
Official call
}

To the officers and members of the American Osteopathic Association:

You are hereby notified that the annual meeting of the American Osteopathic Association will be held July 14 through 19 at the Hyatt Regency Hotel in Dearborn, Mich.

The opening session of the annual meeting of the Board of Trustees will be held at 9 AM on Tuesday, July 14 .

The House of Delegates will convene for the annual business session of the association at 10:30 AM on Friday, July 17 . All meetings of the House of Delegates will be held at the Hyatt Regency Hotel. The Committee on Credentials will register delegates and alternate delegates beginning at $8 \mathrm{AM}$ on Friday, July 17 . The House will conclude its session on Sunday, July 19.

At least 30 days prior to the first day of the annual meeting, the secretary of each divisional society must certify to the executive director of the American Osteopathic Association a list of the names and addresses of delegates and alternate delegates.

GILBERT S. BUCHOLZ, DO President

T. EUGENE ZACHARY, DO Speaker of the House 


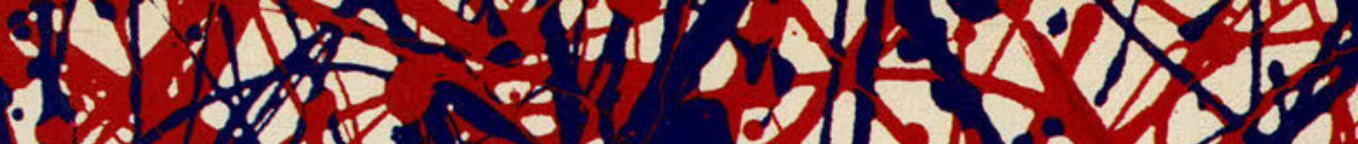




\section{X⿳亠口冋丁口 alprazolami $\mathbb{1}$ \\ For anxiety with associated depressive symptoms}

\section{XANAX Tablets \\ (alprazolam, C Upjohn)}

\section{INDICATIONS AND USAGE}

Generalized anxiety disorder, short-term relief of the symptoms of anxiety, and anxiety associated with depression. Anxiety or tension associated with the stress of everyday life does not usually require an anxiolytic.

Panic disorder, with or without agoraphobia.

Effectiveness has been established for up to 4 months for anxiety disorder and 4 to 10 weeks for panic disorder; but panic disorder has been treated open label for up to 8 months with no apparent loss of benefit. The usefulness should be reassessed periodically.

\section{CONTRAINDICATIONS}

Sensitivity to XANAX or other benzodiazepines and in acute narrow-angle glaucoma.

\section{WARNINGS}

\section{Dependence and withdrawal reactions, including seizures}

Physical dependence on XANAX can produce certain adverse clinical events, some life-threatening. These include a spectrum of withdrawal symptoms-the most important is seizure. Even after relatively short-term use at 0.75 to $4 \mathrm{mg}$ per day, there is some risk of dependence; but the risk and severity seem to be greater in patients treated with doses above $4 \mathrm{mg} /$ day for more than 8 to 12 weeks.

Treatment of panic disorder: Dose and risks of XANAX

Panic-disorder patients often need doses greater than $4 \mathrm{mg} /$ day, hence the risk of dependence may be greater than in patients treated for less severe anxiety. Based on studies involving 641 patients, the most frequent symptoms seen during discontinuation were:

Neurologic: Insomnia, light-headedness, abnormal involuntary movement, headache, muscular twitching impaired coordination, muscle-tone disorders, weakness.

Psychiatric: Anxiety, fatigue and tiredness, irritability, cognitive disorder, memory impairment, depression, confusional state.

Gastrointestinal: Nausea/vomiting, diarrhea, decreased salivation

Other systems: Weight loss, decreased appetite, sweating, tachycardia, blurred vision. It has not been determined if these symptoms are clearly related to dose and duration of therapy. In two controlled trials of the discontinuation of XANAX lasting 6 to 8 weeks, $71 \%$ to $93 \%$ of patients treated with XANAX compared with $89 \%$ to $96 \%$ of placebo patients tapered completely off medication. The ability of patients to completely discontinue after long-term therapy has not been reliably determined. Seizures were reported in 8 of 1,980 patients who took more than $4 \mathrm{mg}$ XANAX daily for over 3 months, generally during abrupt dose reduction. There have been occasional reports of seizures during gradual taper; the risk seems to be greatest

24 to 72 hours after discontinuation.

Status epilepticus and its treatment: Discontinuation of XANAX has been associated with seizures. In most cases a single seizure was reported; however, multiple seizures and status epilepticus have been reported. Treatment is the same as that for status of any cause.

Interdose symptoms: Early morning anxiety and emergence of anxiety symptoms between doses have been reported in panic patients taking XANAX. It can usually be managed with more frequent administration of the same total daily dose or by increasing total dosage.

Risks of dose reduction: Withdrawal reactions may occur with dosage reduction for any reason; dosage should be reduced gradually. (See DOSAGE AND ADMINISTRATION.

XANAX is not of value in treating psychosis and should not be used in lieu of appropriate treatment. Patients receiving XANAX should be cautioned about hazardous occupations or activities requiring full alertness and also about simultaneous ingestion of alcohol or other CNS depressants.

Benzodiazepines can cause fetal harm in pregnant women, hence women who may become pregnant should be warned. Avoid during the first trimester. (See DRUG ABUSE AND DEPENDENCE and DOSAGE AND ADMINISTRATION.)

\section{PRECAUTIONS}

General: If XANAX is combined with other psychotropics or anticonvulsants, consider drug potentiation. (See DRUG INTERACTIONS.) Use the usual precautions in patients with impaired renal, hepatic, or pulmonary function and regarding prescription size in depressed and suicidal patients. Use the smallest effective dose to avoid ataxia or oversedation, which may be a particular problem in elderly or debilitated patients. Alprazolam plasma half-life may be prolonged in obese patients or those with alcoholic liver disease. There have been rare reports of death in patients with severe pulmonary disease shortly after the start of therapy with XANAX. Episodes of mania and hypomania have been reported in depressed patients. Alprazolam is weakly uricosuric

Information for patients: For all users of XANAX. Alert patients about: (1) consumption of alcohol and drugs, (2) not taking any benzodiazepine during pregnancy or while nursing, (3) operating machinery or driving, (4) not increasing the dose due to risk of dependence, (5) not stopping the drug abruptly.

Additional advice for panic patients:

Use of more than $4 \mathrm{mg}$ per day of XANAX for long periods of time may cause severe emotional and physical dependence in some patients, and these patients may find it difficult to stop treatment. In two studies lasting 6 to 8 weeks, $7 \%$ to $29 \%$ of patients treated with XANAX did not completely taper off therapy. The ability of patients to completely discontinue therapy has not been determined. Also, extended use of high doses appears to increase the incidence and severity of withdrawal reactions when XANAX is discontinued. These are generally minor, but seizure, which can be life-threatening, can occur, especially if dose is reduced too rapidly or stopped abruptly.

Laboratory tests: Not ordinarily required in otherwise healthy patients.
Drug interactions: Additive CNS depressant effects with other psychotropics, anticonvulsants, antihistamines, ethanol, and other CNS depressants. Plasma levels of imipramine and desipramine are increased. Pharmacokinetic interactions with other drugs have been reported. Cimetidine and oral contraceptives can delay clearance of benzodiazepines. Drug/laboratory test interactions: No consistent pattern. Carcinogenesis, mutagenesis, impairment of fertility:

No carcinogenic potential or impairment of fertility in rats. Pregnancy: See WARNINGS. Nonteratogenic effects: The child born of a mother on benzodiazepines may be at some risk for withdrawal symptoms, neonatal flaccidity, and respiratory problems. Labor and delivery: No established use. Nursing mothers: Benzodiazepines are excreted in human milk. Women on XANAX should not nurse.

Pediatric use: Safety and effectiveness in children below the age of 18 have not been established.

\section{ADVERSE REACTIONS}

Side effects are generally observed at the beginning of therapy and usually disappear with continued medication. In the usual patient, the most frequent side effects are likely to be an extension of the pharmacologic activity of XANAX, eg. drowsiness or light-headedness.

Central nervous system: Drowsiness, light-headedness, depression, headache, confusion, insomnia, nervousness, syncope, dizziness, akathisia, and tiredness sleepiness. Impaired coordination, irritability, memory impairment, cognitive disorder, dysarthria, anxiety, abnormal involuntary movement, changed libido, muscular twitching, weakness, muscle-tone disorders, agitation, disinhibition, paresthesia, talkativeness, vasomotor disturbances, derealization, dream abnormalities, fear, feeling warm.

Gastrointestinal: Dry mouth, constipation, diarrhea, nausea/vomiting, increased salivation, decreased salivation, and abdominal distress.

Cardiorespiratory: Tachycardia/palpitations, hypotension, nasal congestion, chest pain, hyperventilation, upper respiratory infection.

Other: Blurred vision, rigidity and tremor, tinnitus, muscular stiffness and cramps, sweating, skin disorders, rash, change in appetite, micturition difficulties, menstrual disorders, sexual dysfunction, edema, incontinence, infection, dermatitis/allergy, weight gain, and weight loss

Withdrawal seizures with rapid decrease or abrupt discontinuation. (See WARNINGS.)

To discontinue treatment, dosage must be reduced slowly by no more than $0.5 \mathrm{mg}$ every 3 days.

The following adverse events have been reported with benzodiazepines: dystonia, concentration difficulties, anorexia, slurred speech, jaundice, pruritus, diplopia

Untreated panic disorder has been associated with depressive disorders and suicide. When treating panic patients, the same caution must be used as in treating patients with depression or suicidal ideation.

Paradoxical reactions such as stimulation, agitation, rage, increased muscle spasticity, sleep disturbances, and hallucinations may occur. Should these occur. discontinue the drug

During prolonged treatment, periodic blood counts, urinalysis, and.blood chemistry analysis are advisable. Minor EEG changes, of unknown significance, have been observed

Liver enzyme elevations, gynecomastia, and galactorrhea have been reported; but no causal relationship was established.

\section{DRUG ABUSE AND DEPENDENCE}

Physical and psychologic dependence: Withdrawal symptoms like those noted with sedative/hypnotics and alcohol have been seen after discontinuance of benzodiazepines. Symptoms can range from mild dysphoria and insomnia to a major syndrome including abdominal and muscle cramps, vomiting, sweating, tremors, and convulsions. The distinction between withdrawal symptoms and recurrence of illness is difficult. Withdrawal typically includes new symptoms, occurs toward the end of taper or shortly after discontinuation, and decreases with time. Recurrent panic disorder recurs early or late, with persistent symptoms similar to the initial presentation. When necessary XANAX should be restarted in adequate dosage.

Withdrawal symptoms, including seizures, may occur after brief therapy with dosages of 0.75 to $4 \mathrm{mg} /$ day, but severity and incidence are apparently increased after dosages above $4 \mathrm{mg} /$ day, after rapid decrease of dosage or abrupt discontinuation. Dosage should be gradually tapered under close supervision, especially in patients with a history of seizures or epilepsy.

Psychologic dependence is a risk with all benzodiazepines, increasing at higher doses, with long-term use, and in patients with a history of alcohol or drug abuse. Addiction-prone patients should be closely supervised when receiving XANAX and repeat prescriptions limited.

Controlled Substance Class: XANAX is a controlled substance and has been assigned to schedule IV.

\section{OVERDOSAGE}

Manifestations include somnolence, confusion, impaired coordination, diminished reflexes, and coma. Death has been reported with overdosage of benzodiazepines by themselves or in combination with overdosage of alcohol.

\section{DOSAGE AND ADMINISTRATION}

Dosage should be individualized using the lowest effective dose. The need for treatment should be reassessed frequently.

Anxiety: The usual starting dosage is 0.25 to $0.5 \mathrm{mg}$ ti.d. Maximum daily dose is $4 \mathrm{mg}$. Patients who are elderly or debilitated, or have advanced liver disease, may be especially sensitive to benzodiazepines, and the starting dosage is $0.25 \mathrm{mg}$ b.i.d. or ti.d. Reduce dosage gradually, by no more than $0.5 \mathrm{mg}$ every 3 days, or more slowly.

Panic disorder: Many patients required more than $4 \mathrm{mg}$ daily. Mean dosage in studies was 5 to $6 \mathrm{mg} /$ day, with a maximum of $10 \mathrm{mg} /$ day. Treatment may be initiated with $0.5 \mathrm{mg}$ ti.d. Depending on the response, dosage may be increased every 3 or 4 days by up to $1 \mathrm{mg}$ daily. After extended freedom from attacks, dosage should be carefully tapered by, at most, $0.5 \mathrm{mg}$ every 3 days. Some patients may be resistant to all discontinuation regimens.

HOW SUPPLIED

XANAX Tablets are available as 0.25-mg, 0.5-mg, 1-mg, and 2-mg tablets

CAUTION: FEDERAL LAW PROHIBITS DISPENSING WITHOUT PRESCRIPTION

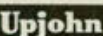

HE UPJOHN COMPANY
January 1992 